\title{
DOMINANT COLOR STRUCTURE DESCRIPTOR FOR IMAGE RETRIEVAL
}

\author{
Ka-Man Wong, Lai-Man Po, and Kwok-Wai Cheung \\ kmwong@ee.cityu.edu.hk, eelmpo@cityu.edu.hk, kwcheung@ee.cityu.edu.hk \\ Department of Electronic Engineering, City University of Hong Kong, \\ 83 Tat Chee Avenue, Kowloon, Hong Kong SAR of China.
}

\begin{abstract}
A new Dominant Color Structure Descriptor (DCSD) is proposed in this paper. It is designed to provide an efficient way to represent both color and spatial structure information with single compact descriptor. The descriptor combines the compactness of Dominant Color Descriptor (DCD) and the retrieval accuracy of Color Structure Descriptor (CSD) to enhance the retrieval performance in a highly efficient manner. The feature extraction and similarity measure of the descriptor are designed to address the problems of the existing descriptors while utilize the advantages of them. Experimental results show that DCSD has a significant improvement on both retrieval performance and descriptor size over DCD. An eight-color DCSD (DCSD 8) gives an Averaged Normalized Modified Retrieval Rate (ANMRR) of 0.0993 using MPEG-7 common color dataset, outperforming compact configurations of Scalable Color Descriptor and Color Structure Descriptor with smaller descriptor size.
\end{abstract}

Keywords - Content Based Image Retrieval, Dominant Color Structure Descriptor.

\section{INTRODUCTION}

Recently, the rapid development of digital image and video devices catalyzed the growth of digital multimedia contents. In response to the need of efficient management of multimedia database systems, MPEG-7 [1] has been developed as a standard for multimedia content description to facilitate interoperability between different searching engines and different databases for multimedia data searching and content exchange. For describing multimedia contents, low level features are usually used since they can be generated efficiently. Among the low level image features, color is the most expressive and effective in visual content description. Several color descriptors [2] have been defined in MPEG-7 for describing color features of multimedia contents. These color descriptors are applicable to a broad range of applications according to their complexity (in terms of extraction and similarity measure) and efficiency (in terms of descriptor size and accuracy).

Among the color descriptors, Dominant Color Descriptor (DCD) [2], is a compact color descriptor, it stores the dominant colors, their percentages, and several optional parameters. Since DCD stores only the dominant colors (max. 8 colors) instead of a color histogram, the storage requirement is very effective with relatively small redundancy. Unfortunately, DCD performance is the worst among various color descriptors in its existing usage as reported in [2-3]. Its performance is even worse than a more compact descriptor, the Color Layout Descriptor (CLD). The performance of DCD is quite limited due to the lack of spatial color distribution information and the color accuracy problem. Among various MPEG-7 color visual descriptors, Color Structure Descriptor (CSD) always gives the best retrieval rate. The main reason is the capture of spatial color distribution information in CSD. However, CSD have redundancy as it uses a fixed color space for the histogram representation. The most compact CSD configuration takes about 32 bytes for each image. The size is approximately 4 times that of CLD and about double of DCD. This might be negligible but, for mobile or other bandwidth concerned applications, every bit should be saved. To address this problem we can combine the dominant color features of DCD and the spatial color distribution structure of CSD to design a new color descriptor, called Dominant Color Structure Descriptor (DCSD), which maintains the compactness of dominant colors while significantly improving the retrieval accuracy by using structured dominant color histograms for introducing spatial distribution information of the image.

The organization of the remaining sections of this paper is as follows. Section II gives an overview of DCD and CSD which forms the basis for the subsequent discussion. Section III describes the core of the proposed descriptor - dominant color structure histogram. Section IV presents the similarity measure developed for DCSD. Finally, experimental results and a conclusion are presented in Section V and Section VI, respectively.

II. LimitaTIONS OF DOMINANT COLOR DESCRIPTOR AND COLOR STRUCTURE DESCRIPTOR

\section{A. Dominant Color Descriptor}

With target application in similarity retrieval in large image database, dominant color descriptor extracts the features from an image by clustering the colors in an image into a small number of colors and is defined as

$$
F=\left\{\left(c_{i}, p_{i}, v_{i}\right), s\right\},(i=1, \ldots, N) .
$$

The descriptor consists of the representative colors $c_{i}$, their percentages $p_{i}$, the optional color variances for each dominant color $v_{i}$, and the optional spatial coherency $s$ of the dominant colors. The quadratic histogram distance measure (QHDM) [2] is used for similarity measure for DCD. With this simple and compact representation, DCD allows efficient indexing for similarity retrieval while sacrificing retrieval accuracy due to lack of spatial information of the description compared to other color descriptors. In DCD feature extraction, Generalized Lloyd Algorithm (GLA) [4] color quantization is applied on the image. Since GLA is a splitting process based on minimizing distortion of each cluster, the colors obtained by GLA can be very close. However, QHDM distance measure requires that the colors in a descriptor should have values with at least $T_{d}$ away to maintain the metric of the similarity measure [2]. Thus, after the GLA quantization, agglomerative clustering is applied to the quantized colors by searching the closest pair of colors, $c_{1}$ and $c_{2}$ in CIELuv color space. Then these two colors will be merged together based on liner combination of their percentages $p_{1}$ and $p_{2}$. The merging process iterates until the minimum internal distance is larger than the threshold $T_{d}$ so as to comply with the requirement of QHDM. The distortion due to the GLA process will not be reduced as this clustering process is not a reverse operation of GLA. Also, the additional distortion 
introduced is not minimized since the merging is not based on distortion minimization. Thus, the clustering makes the mixed colors become more inaccurate and affect the retrieval performance. Although alternative algorithms are developed for DCD and have improvements $[5,6]$, the performance is still limited.

\section{B. Color Structure Descriptor}

Color structure descriptor [2] is based on color histogram, but aims at providing a more accurate description by identifying localized color distributions of each color. CSD is characterized by a color structure histogram for $M$ quantized color, $c_{m}$, and is expressed as

$$
h(m), m=1, \ldots, M
$$

where $M \in\{256,128,64,32\}$ and the bin value $h(m)$ is the number of structuring elements containing one or more pixels with color $c_{m}$. Unlike the conventional histogram, the color structure histogram is extracted from an image by accumulation using an $8 \times 8$-structuring window. The structuring element scans the image and counts the number of times a particular color is contained with the structuring element. Denote $I$ be the set of quantized color index of an image and $S \in I$ be the set of quantized color index existing inside the subimage region covered by the structuring element. With the structuring element scanning the image, the color histogram bins are accumulated according to

$$
h(m) \leftarrow h(m)+1, \quad m \in S
$$

Thus, the final value of $h(m)$ is determined by the number of positions at which the structuring element contains $c_{m}$.

An $L 1$ distance measure is used to compute the dissimilarity between CSDs. CSD provides more accurate similarity retrieval because of the inclusion of spatial color information. This representation is more closely related to the human perception and, thus, is more useful for indexing and retrieval. Structure histogram describes color feature very well and can give very high retrieval accuracy.

Although the color structure histogram contributes to the high retrieval accuracy of CSD, the fixed color space requirement of the histogram results in redundancy in the representation. For example, a DCD with eight colors need 21.75 bytes in binary representation, and a DCD with 4 colors only need 11.75 bytes. In the contrary, the most compact CSD 32 uses 32 bytes per descriptor, which is about 1.5-3 times of DCD. In the following section, a new dominant color structure descriptor is proposed to achieve both compact descriptor size of DCD and relatively high retrieval accuracy of CSD.

\section{Dominant Color Structure Histogram}

The proposed DCSD combines the advantages of DCD and CSD, but there are fundamental differences between DCD and CSD prevent us from directly apply particular techniques from one descriptor to another. The first difference is the color feature space. DCD uses a quantized colors space while CSD uses a statically partitioned HMMD color space. As a result, DCD requires the similarity measure algorithm consider the similarity between colors while CSD only need a simple $L 1$ distance for the similarity measure. Another difference is the representation of the percentages in DCD and structure histogram bins in CSD, percentages directly proportional to the number of pixel thus the meaning of summation of percentages is very clear. On the contrary, summation of structure histogram bin does not represent the area covered by the colors since there may be overlapping between them. As a result, color merging techniques used in variable color space like DCD cannot be directly applied on color structure histograms. In order to tackle these problems, the two processes for generating of the proposed dominant color structure histogram will be described below.

\section{A. Color Quantization}

The number of quantizing colors and the descriptor size are limited by the maximum number of clusters allowed in GLA splitting. To maintain the minimum distortion achieved by GLA, no agglomerative clustering is applied to the resulted clusters as in DCD. As a result, there is no restricted minimum distance between quantizing colors.

Given a set of quantizing colors $C_{q}=\left\{c_{1}, c_{2}, \cdots, c_{n}\right\}$ obtained from GLA, a closest quantizing color,

$$
c_{\min }=c_{i} \mid \min \left(d\left(c_{x y}, c_{i}\right)\right) ; c_{i} \in C_{q}
$$

is found for an image pixel with color value $c_{x y}$ at location $(x, y)$. The pixel is quantized to

$$
c_{x y}=\left\{\begin{array}{lc}
c_{\min } & \text { if } d\left(c_{x y}, c_{\min }\right)<T_{d} \\
\text { null } & \text { otherwise }
\end{array}\right.
$$

where $d\left(c_{x y}, c_{\min }\right)$ is the distance between the original color and closest dominant color. The threshold $T_{d}$ can be in the range of 15 20 without significant difference and $T_{d}=20$ is selected in our experiments. It should be noted that this threshold is used only for quantizing image. The dominant color set is not changed at all. The color of each pixel $c_{x y}$ is quantized to the closest color $c_{\min }$ in the dominant colors set if the color distance $d\left(c_{i j}, c_{\text {min }}\right)$ between the original pixel and the closest dominant color is smaller than a threshold $T_{d}$ in the CIELuv color space. Otherwise, the pixel will be ignored. Some pixels are ignored because GLA only minimizes the overall distortion but not the distance between dominant colors. Some pixels might not be similar to any dominant colors. Thus, such pixels are not considered in the feature extraction as they are irrelevant with the dominant colors.

\section{B. Structure Block Scanning}

In the proposed method, color structure bins is formed by accumulation using an $8 \times 8$ structuring element. In each structure block scanning, the structuring element scans through every pixel position of the image while keeping the entire structuring element within the image. A set of color bins is maintained for the scanning. At each pixel position, the colors existed in the region enclosed by the structuring element are recorded by the increasing the corresponding color bin count by one. A histogram will be formed with the bin values counting the times that the structuring element enclosed the colors in the scanning. The histogram will then be normalized by the number of pixel positions that the structuring element scanned. It should be noted that each bin is being normalized. It is different from conventional histogram that the histogram sum is being normalized. This leads to a significant difference in the interpretation of the bins. Each color structure histogram bin represents the area covered by the color in the image while, in the conventional histogram, each bin represents the number of occurrence of the color in the image. Figure 1 illustrates the DCSD extraction procedures.

\section{Differences between DCSD and DCD and CSD}

From the extraction process described above, DCSD has significant differences from CSD and DCD. As CSD uses a fixed color space for counting the histogram, the color set of the histogram will be the same and a simple $L 1$ distance can be used for similarity measure, but it is not suitable for variable color space like DCSD since it may not have exactly matched dominant colors between two images. In DCD, dominant colors are computed in extraction process. Each image may have their own dominant color set. DCD uses a quadratic-like similarity measure, which is based on an assumption that the sum of the percentages is normalized. As a different normalization method is used in the structure histogram, it not suitable to directly apply the original DCD similarity measure 
on DCSD. Therefore, a new similarity measure for the structure histogram is proposed in the next section.

\section{Color Matching Palette Similarity Measure \\ The proposed descriptor, DCSD, is defined as}

$$
F=\left\{\left(c_{i}, q_{i}\right), s\right\} \quad \text { for } i=\{1,2, \cdots, N\} .
$$

It consists of the number of dominant colors $(N)$, color values $\left(c_{i}\right)$ structure values of dominant colors $\left(q_{i}\right)$, and with the optional spatial coherency parameter $(s)$. The major difference between DCSD and DCD is that, in DCSD, $q_{i}$ represents color structure bins instead of color percentage. Each $q_{i}$ is normalized instead of the sum of $q_{i}$ is normalized. It is done by a new extraction method proposed. DCSD inherits the compactness of DCD. For example, a DCD with eight colors requires 21.75 bytes in binary representation, and a DCD with 4 colors requires only 11.75 bytes. The most compact CSD, however, needs 32 bytes.

Similarity measure methods $[5,6]$ proposed for DCD perform better than the original QHDM similarity measure. As all these methods involve splitting or merging the colors and their percentages, they may not be suitable for DCSD. In DCSD, the percentages represent the color structure properties and the meaning of merging or splitting two or more color structure bins are fundamentally different from that of conventional color histogram. Thus, a new similarity measure, Color Matching Palette Similarity Measure (CMPSM), which uses color matching based on the color distance between colors, is developed for DCSD.

The DCSDs $F_{1}, F_{2}$ from two matching images are defined as

$$
\begin{array}{ll}
F_{1}=\left\{\left(c_{1 i}, q_{1 i}\right), s_{1}\right\} & \text { for } i=\left\{1,2, \cdots, N_{1}\right\} \\
F_{2}=\left\{\left(c_{2 j}, q_{2 j}\right), s_{2 j}\right\} & \text { for } j=\left\{1,2, \cdots, N_{2}\right\}
\end{array}
$$

In the matching process, pairs of matching colors with the minimum color distance and with within $T_{d}$ distance are picked up from the color set of the two DCSD descriptors. The color distances for these minimum distance pairs are denoted as $c d_{i}$ are added to a color matching palette. The color distance is measured by the Euclidian distance between the matched DCSD colors in CIE $L u v$ color space. Also as part of the palette is the structure differences $s d_{i}$ defined as the absolute difference between the structure bins of the corresponding minimum distance pairs. This process iterates until no more similar color pairs can be found. Accordingly, the Color Matching Palette is given as

$$
P_{m}=\left\{s d_{i}, c d_{i}\right\} \text { for } i=1, \ldots, N_{m} .
$$

After that, the maximum matching color distance $c d_{\max }$ in $c d_{i}$ is found. As some colors may not be matched for descriptors with different number of dominant colors, the unmatched colors are appended to the matching palette by setting $s d_{i}$ equals as its original structure bin $q_{i}$, and $c d_{i}$ as the maximum matching distance $c d_{\max }$. The distance between two DCSD descriptors is defined as

$$
D\left(F_{1}, F_{2}\right)=1-\sum_{i}\left[\left(1-s d_{i}\right)\left(1-\frac{c d_{i}}{\beta T_{d}}\right)\right]
$$

where $\beta$ is a parameter used to adjust the importance of color distance. Setting $\beta=\infty$ means that the color distance is not considered. And setting $\beta=1$, which is the minimum value of $\beta$ means that the importance of color distance and color structure are equal. By trying with different values of $\beta$, a range of $2<\beta<5$ is suitable for matching most natural images. The result of $D\left(F_{1}, F_{2}\right)$ is between 0 and 1 , where smaller values represents more similar between images.

\section{EXPERIMENTAL RESULTS}

The proposed method is implemented in a CBIR system, MIRROR [7]. The descriptor extraction and searching are based on MPEG-7

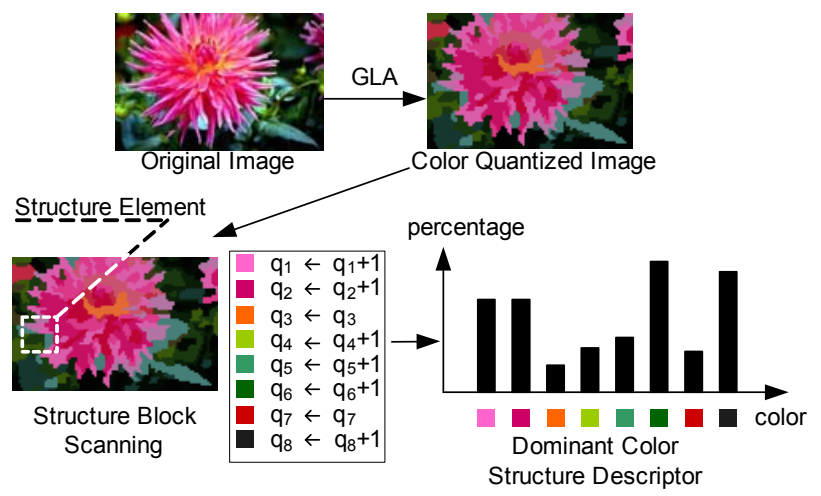

Fig. 1 Dominant Color Structure Descriptor extraction

reference software XM with modifications. In our experiments, we use the Common Color Dataset (CCD) in MPEG-7 core experiments [8]. The dataset contains 5466 images and a common ground truth set with 50 sample queries defined. All descriptors are encoded with MPEG-7 binary format by XM.

Averaged Normalized Modified Retrieval Rate (ANMRR) and Normalized Modified Retrieval Rate (NMRR) [9] are used to measure the performance of retrieval. To measure the performance of the proposed method, several configurations of DCSD with 4 to 8 colors with $\beta=5, T_{d}=20$ is used in the experiments. The proposed DCSD is compared with the color descriptors included in the current XM 6.1 including Dominant Color Descriptor with the original similarity measure (DCD-QHDM), Color Structure Descriptor (CSD), Scalable Color Descriptor (SCD) and Color Layout Descriptor (CLD). In addition we also compared with the DCD with Merged Palette Histogram Similarity Measure (DCDMPHSM) [5]. It should be noted that all DCD and DCSD results are with the optional spatial coherency parameter enabled.

\section{A. Ultra Compact Configurations}

Using ultra compact configurations, DCSD outperforms DCD in both retrieval accuracy and descriptor size. TABLE I shows that DCSD 4 and DCSD 5 have less than 16 bytes per descriptor. DCSD can give an acceptable retrieval result. Although DCSD can use even fewer colors to make the descriptor more compact, it is not possible to satisfactorily describe an image with such small number of colors. Although DCSD 4 gives ANMRR of 0.2371, which is not the best among the ultra compact descriptors and does not perform better than a more compact CLD, it already achieves a retrieval result very close to the original DCD having 0.2328 ANMRR but with a descriptor size smaller by $27.74 \%$. DCSD 5 gives an ANMRR of 0.1878 , which is one of the best retrieval rates among the ultra compact descriptors. DCSD 5 is also very competitive with the best ultra compact SCD configurations such as $\operatorname{SCD}(16,2)$, $\operatorname{SCD}(64,4), \operatorname{SCD}(16,0), \operatorname{SCD}(32,3)$, where, in $\operatorname{SCD}(\mathrm{x}, \mathrm{y}), \mathrm{x}$ is the number of colors and $\mathrm{y}$ is the number of bit-planes discarded for each histogram bin. Based on the above analysis, CLD, DCSD 5, and $\operatorname{SCD}(16,2)$ are good choices for the ultra compact color descriptors.

\section{B. Size-accuracy Configurations}

From TABLE I, DCSD 8 gives an ANMRR of 0.0993, which is the best among the descriptors in this range. It outperforms the most accurate $\operatorname{SCD}(64,2)$ by $18.54 \%$ with descriptor size smaller by $25 \%$. DCSD 8 also slightly outperforms CSD 32 by $2.55 \%$ with descriptor size substantially smaller by $32 \%$. Some visual results comparison between DCSD 5, DCSD 8, DCD QHDM and CSD 32 are also given in Figure 2 in which DCSD 5 and DCSD 8 retrieved more visually similar images than CSD and DCD QHDM. TABLE 
I also shows that DCSD outperforms every configuration of SCD with the same descriptor size. For examples, DCSD 6, DCSD 7 and DCSD 8 outperform $\operatorname{SCD}(128,8), \operatorname{SCD}(32,2)$ and $\operatorname{SCD}(128,4)$ by $6.78 \%, 14.65 \%$ and $19.73 \%$, respectively. Generally, DCSD is more accurate than SCD with same descriptor size.

TABLE I: Results of MPEG-7 color descriptors

\begin{tabular}{|c|c|c|c|}
\hline & Descriptor & $\begin{array}{l}\text { Avg. Descriptor } \\
\text { Size (bytes) }\end{array}$ & ANMRR \\
\hline \multirow{9}{*}{ 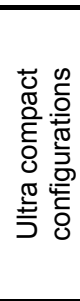 } & CLD & 8.00 & 0.2264 \\
\hline & $\overline{\operatorname{SCD}(64,8)}$ & 8.75 & 0.2511 \\
\hline & $\operatorname{SCD}(16,3)$ & 9.50 & 0.2108 \\
\hline & $\operatorname{SCD}(16,2)$ & 11.50 & 0.1928 \\
\hline & DCSD 4 & 11.75 & 0.2371 \\
\hline & DCSD 5 & 14.25 & 0.1878 \\
\hline & $\operatorname{SCD}(64,4)$ & 15.00 & 0.1816 \\
\hline & $\operatorname{SCD}(16,0)$ & 15.50 & 0.1813 \\
\hline & $\overline{\operatorname{SCD}(32,3)}$ & 16.00 & 0.1826 \\
\hline \multirow{15}{*}{ 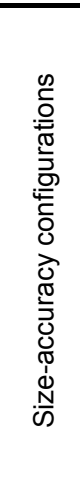 } & DCD-QHDM & 16.26 & 0.2328 \\
\hline & DCD-MPHSM & 16.26 & 0.2297 \\
\hline & $\overline{S C D}(128,8)$ & 16.75 & 0.1648 \\
\hline & DCSD 6 & 16.75 & 0.1536 \\
\hline & $\operatorname{SCD}(32,2)$ & 18.50 & 0.1481 \\
\hline & DCSD 7 & 19.25 & 0.1264 \\
\hline & $\operatorname{SCD}(64,3)$ & 21.00 & 0.1583 \\
\hline & DCSD 8 & 21.75 & 0.0993 \\
\hline & $\overline{\operatorname{SCD}(128,4)}$ & 23.00 & 0.1237 \\
\hline & $\overline{\operatorname{SCD}(32,0)}$ & 26.50 & 0.1367 \\
\hline & $\operatorname{SCD}(64,2)$ & 29.00 & 0.1219 \\
\hline & $\operatorname{SCD}(128,3)$ & 31.00 & 0.1278 \\
\hline & CSD 32 & 32.38 & 0.1019 \\
\hline & $\overline{\operatorname{SCD}(256,8)}$ & 32.75 & 0.1516 \\
\hline & $\operatorname{SCD}(256,6)$ & 33.75 & 0.1527 \\
\hline
\end{tabular}

\section{Conclusions}

An efficient color structure, dominant color structure histogram, for color image representation is described. A new color descriptor, Dominant Color Structure Descriptor (DCSD), based on the structure histogram is developed for color image retrieval. The descriptor inherits the compactness of Dominant Color Descriptor (DCD) and the retrieval accuracy of Color Structure Descriptor (CSD). The new similarity measure algorithm based on matching similar colors to generate a common palette, instead of using a fixed histogram space, is developed for DCSD. Experiment results show that DCSD gives better retrieval performance than the original DCD. Moreover, the effective configuration, DCSD 8, outperforms the most accurate SCD and CSD configurations with even smaller descriptor size. With its compactness and accuracy properties, DCSD is highly competitive as compared with the current color descriptors in a wide range of descriptor sizes.

\section{Acknowledgment}

The work described in this paper was substantially supported by a grant from City University of Hong Kong, Hong Kong SAR, China. [Project No.7001853].

\section{REFERENCES}

[1] Special issue on MPEG-7, IEEE Trans. Circuits and Systems for Video Technology, vol.11, no. 6, June 2001.

[2] B.S. Manjunath, J. R. Ohm, V. V. Vasudevan, A. Yamada, "Color and texture descriptors," IEEE Trans. on Circuits and Systems for Video Technology, vol. 11, no. 6, 703 -715, June 2001.

[3] T. Ojala, M. Aittola, and E. Matinmikko, "Empirical evaluation of MPEG-7 XM color descriptors in content-based retrieval of semantic image categories," in Proc. of Int'l Conf. on Pattern Recognition, vol. 2, pp. 1021-1024, August 2002.
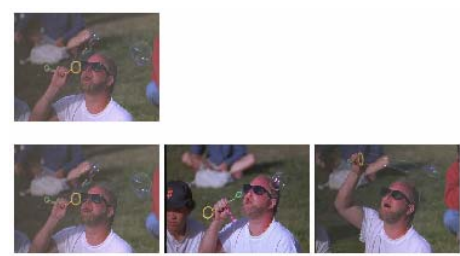

Fig. 2a Reference image and ground truth set of query \#48

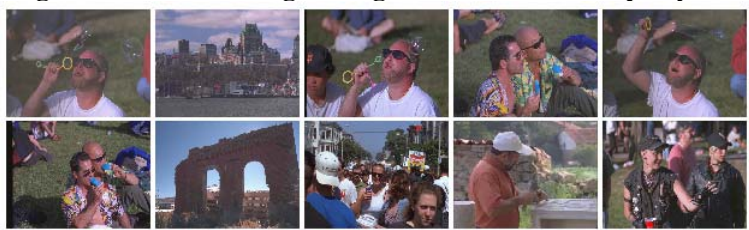

Fig. 2b Visual result of DCSD 5, NMRR $=0.0769,3$ of 3 ground truth images found in the first 10 retrievals.

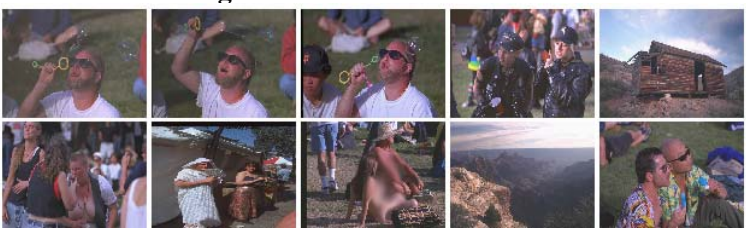

Fig. 2c Visual result of DCSD 8, NMRR $=0.0000,3$ of 3 ground truth images found in the first 10 retrievals.

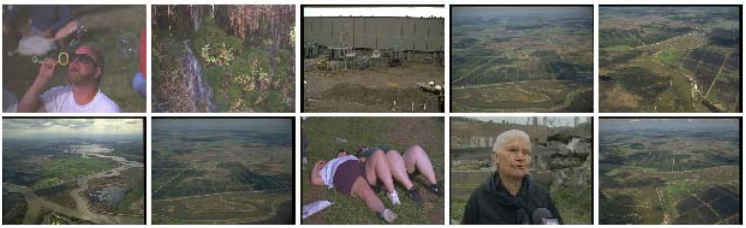

Fig. 2d Visual result of DCD QHDM, NMRR $=0.6410,1$ of 3 ground truth images found in the first 10 retrievals.

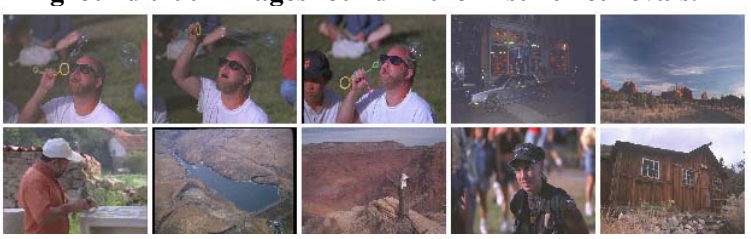

Fig. 2e Visual result of CSD 32, NMRR $=0.0000,3$ of 3 ground truth images found in the first 10 retrievals.

[4] A. Gersho and R.M. Gray, "Vector Quantization and Signal Compression," Kluwer Academic Publishers, 1992.

[5] L. M. Po and K. M. Wong, "A new palette histogram similarity measure for MPEG-7 Dominant Color Descriptor," in Proc. of IEEE Int'l Conf. on Image Processing 2004, vol. 3 pp. 1533-1536. 24-27 Oct. 2004, Singapore.

[6] J. Chen, T. N. Pappas, A. Mojsilović and B. E. Rogowitz, "Adaptive perceptual color-texture image segmentation," IEEE Trans. on Image Processing, vol. 14, no. 10, October 2005. pp. 1524-1536.

[7] K. M. Wong, K. W. Cheung and L. M. Po, "MIRROR: an interactive content based image retrieval system," in Proc. of IEEE Int'l Symposium on Circuit and Systems 2005, vol. 2, pp. 1541-1544. 23-26 May 2005, Kobe, Japan. (http://www.ee.cityu.edu.hk/ mirror)

[8] D. Zier and J. R. Ohm, "Common Data Sets and Queries in MPEG-7 Color Core Experiments," ISO/IEC JTC1/SC29/WG11 Doc. M5060, October 1999.

[9] P. Ndjuki-Nya et al., "Subjective Evaluation of the MPEG-7 Retrieval Accuracy Measure (ANMRR)," ISO/WG11, Doc. M6029, May 2000. 